\title{
Mechanically controllable break-junctions for use as electrodes for molecular electronics
}

\author{
T Böhler ${ }^{1}$, J Grebing ${ }^{1}$, A Mayer-Gindner ${ }^{2}$, H v Löhneysen ${ }^{2,3}$ and \\ E Scheer $^{1,4}$ \\ ${ }^{1}$ Fachbereich Physik, Universität Konstanz, Universitätsstraße 10, D-78464 Konstanz, \\ Germany \\ ${ }^{2}$ Physikalisches Institut, Universität Karlsruhe, D-76128 Karlsruhe, Germany \\ ${ }^{3}$ Forschungszentrum Karlsruhe, Institut für Festkörperphysik, D-76021 Karlsruhe, Germany \\ E-mail: elke.scheer@uni-konstanz.de
}

Received 30 December 2003

Published 17 May 2004

Online at stacks.iop.org/Nano/15/S465

DOI: $10.1088 / 0957-4484 / 15 / 7 / 054$

\begin{abstract}
We discuss the use of mechanically controllable break-junctions (MCBs) as electrodes for contacting individual objects such as clusters or molecules. A big advantage of MCB electrodes is the possibility of varying the contact geometry and, accordingly, the contact resistances and the quantum coherent transport properties between the object under study and the electrodes. We compare the suitability of single-atom contacts of different elements as electrode material. Finally, we present preliminary results on electronic transport through individual (or a few) fullerene molecules contacted by gold MCB electrodes. The typical resistances are in the order of the quantum of conductance. The circuits sustain voltages of the order of $1 \mathrm{~V}$ and are mechanically more stable than single-atom contacts between the gold electrodes alone.
\end{abstract}

\section{Introduction}

To study the electronic transport through individual objects such as small clusters or molecules, electrodes are required that are separated by the typical size of the object under study. With standard e-beam lithography, electrode pairs with spacings below approximately $20 \mathrm{~nm}$ are difficult to define reproducibly. Therefore, several alternative methods, including electromigration methods [1] or electro-deposition [2], have been proposed and used successfully for special applications. However, the success rate is also usually rather low with these methods. Another drawback of using electrodes that are fixed on a substrate is the lack of flexibility of the contact configuration once the molecule of interest has been contacted. Joachim et al [3] have used a scanning tunnelling microscope (STM) for studying the electronic transport through a single C60 fullerene molecule. In the seminal experiment by Reed et al [4] it has been demonstrated that individual molecules can

4 Author to whom any correspondence should be addressed. be contacted with the help of (MCB) techniques, mechanically controlled breakjunctions, stimulating further work $[5,6]$. Simultaneously, very powerful theoretical and computational methods including density functional approaches and Green function techniques have been developed. (For a recent overview of this fast developing field, see e.g. [7].)

However, there is usually a large discrepancy between the calculated and measured conductance value of a system consisting of a pair of electrodes and, say, a molecule in between, even if the calculated and experimental shapes of its current-voltage characteristic $(I-V)$ are in reasonable agreement [5]. There are several possible reasons for this observed quantitative disagreement. A first problem is the determination of the number of molecules contributing to the current. Indirect evidence of the number of objects can be gained by the combination of variation of electrode distance, symmetry arguments [6] or more sophisticated nonlinear transport properties [8]. Second, in the experiments not much is known about the electrode geometry on the atomic scale. The same holds for the contact resistance between 
the electrodes and the molecule. Even if the resistance of the thin film comprising the contact is obtained from an independent measurement, it does not determine the quantum properties of the contacts. Important parameters are, among others, the nature of the electronic transport in the electrodes (e.g. diffusive versus ballistic, coherent versus incoherent), the number of transport channels of the electrodes and the contacts, the transmission coefficients of those channels andfor magnetic materials - the spin polarization of the transport current.

A crucial problem that most of the contacting methods have in common is the lack of knowledge about the precise atomic arrangement of the closest point. To the best of our knowledge, at present there is no solution to this problem in sight. Information about the contact geometry is achieved indirectly by comparison between the experimental findings and a theoretical model of the circuit. Thus, the characterization of the quantum properties of the electrodes is a prerequisite of reliable calculations.

In order to limit the number of degrees of freedom that have to be considered in the calculations, the quantum properties of the electrodes should be simple. It is one of the objectives of this paper to discuss how electrodes can be characterized and which materials are suitable as contact materials from this point of view.

\section{Transport channels}

The conductance and further transport properties of a quantum coherent structure can be characterized by a set of independent transport channels and their transmission values. This concept has been introduced by Landauer [9] and extended to more complex structures by Büttiker [10]. It describes the electronic transport in terms of independent modes of the electronic wavefield, i.e. the transport channels. (For a comprehensive introduction to the field see, e.g., [11].) The electrical conductance of a quantum-coherent structure accommodating $N$ channels is given by the Landauer formula $G=$ $G_{0} \sum_{n=1}^{N} \tau_{n}$, where $G_{0}=2 e^{2} / h$ is the conductance quantum ( $e$ is the electron charge and $h$ is Planck's constant) [9], and $\tau_{n}$ is the transmission probability of the $n$th channel. The $\tau_{n}$ may adopt values between zero and one: $0<\tau_{n} \leqslant 1$. Measuring the conductance is thus equivalent to measuring the $\operatorname{sum} \sum_{n=1}^{N} \tau_{n}$.

For certain applications in molecular electronics it might be advantageous not only to make use of the smallness of the object, resulting e.g., in large charging energies [12], but to profit from quantum-coherent transport properties as well. In order to enhance quantum-coherent transport between the electrodes and the nano-object, it is favourable to have a small number of highly transmissive channels rather than tunnel contacts with a large number of channels with very small transmission or diffusive leads with a more complicated distribution of the transmission values [13].

One of the simplest systems of molecular electronics consists of one well characterized nano-object connected to two electrodes via one channel on each side. As mentioned above, it is possible to contact individual objects with MCB electrodes. The contacts are formed by a single atom or a few atoms each. Depending on the nature of the molecule and the electrode metal the contact might be built by a strong chemical bond or weak van-der-Waals type interaction. Thus, in order to find a suitable electrode material it is necessary to study the structural properties, i.e. the possibility of forming atomically sharp tips, and the conduction properties of the electrodes, as well as the chemical properties, i.e. the nature of the bond with the object under study and the possibility of preparing atomically surfaces.

\section{Characterization of the transport properties of the electrodes}

Consequently, the characterization of the electrodes comprises several parts. First the formation and stability of single-atom wide tips is discussed.

As has been shown previously [14], the conductance varies stepwise when a metallic nanobridge is elongated. The step height is of the order of $G_{0}$ and the average step length corresponds roughly to the lattice constant, i.e. the size of an atom in the solid. For certain metals, e.g. Au, Pt and Ir, the tendency to form longer (last) steps has been shown to be an indication of the formation of one or few atoms wide chains [14-16]. At a certain point of the elongation the conductance starts to evolve exponentially with the distancea clear indication of vacuum tunnelling and hence the fact that the bridge has been broken. Since for every experiment the constriction atoms arrange differently, the opening traces vary from run to run when cycling the distance. The typical behaviour of a metal is studied by repeated opening and closing of the contact and subsequent construction of a histogram of adopted conductance values. One of the best studied elements in this context is the noble metal Au. Although the published histograms achieved by different experimental techniques including STM, 'dangling wire' [17] and MCB deviate slightly in detail, there is agreement about the preferred conductance value of the first peak of the histogram. This value is assumed to correspond to the single-atom contact and is very close to $1 G_{0}$ [14]. These observations demonstrate that $\mathrm{Au}$ can form atomically fine contacts and wires.

Most of the histograms are constructed from so-called opening curves, i.e. when decreasing the conductance. However, for contacting nano-objects it is more important to investigate the probability to form atomically sharp tips by analyzing closing curves, i.e. when approaching the initially separated electrodes and increasing the conductance. Systematic studies with lithographic break-junctions demonstrate that the histograms for closing and opening differ from each other for gold contacts. Figure 1 displays typical closing and opening curves recorded for lithographic break-junctions at very low temperatures $T<100 \mathrm{mK}$ and under cryogenic vacuum conditions, and histograms constructed from these data. (Due to the slow mechanical drive it is not possible to record more than about 100 curves within a day.)

Several observations are remarkable. The steps of the individual openings are not well pronounced, while for closing curves they are usually clearly recognizable. The histogram peaks, both for opening and closing, are broad and shifted to smaller conductances compared to the observations for samples fabricated with different techniques (STM or 'notched-wire' breakjunctions) [14]. We attribute this difference to the creation of defects during the plasma 


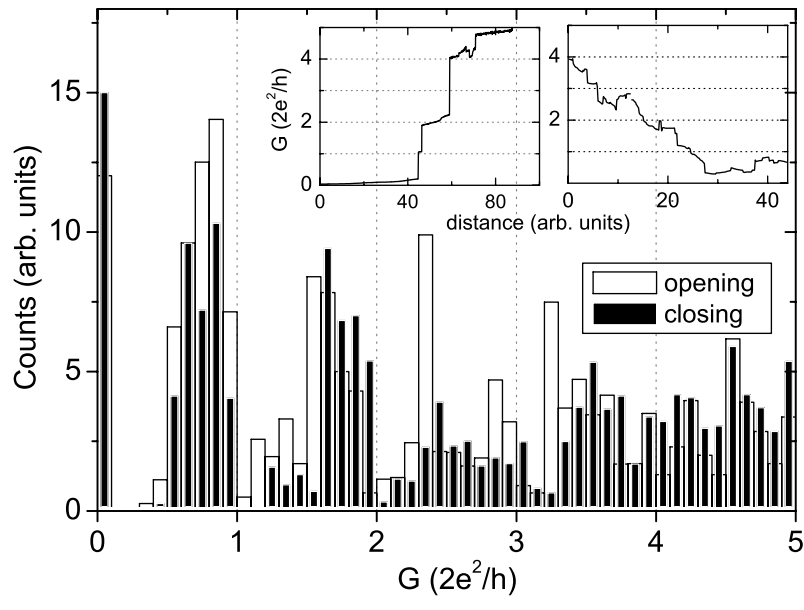

Figure 1. Conductance histogram of Au lithographic break-junctions, measured at $T<100 \mathrm{mK}$, constructed from $\sim 100$ individual opening and closing curves, respectively. Insets: individual opening (right) and closing (left) curves.

etch process that is used to suspend the nanobridge [18]. Second, the first peak in the closing histogram is much lower than in the opening histogram. We interpret this as the tendency towards bigger than single-atom contacts as first contacts when approaching the electrodes, as suggested by molecular dynamics simulations [19], and directly observed in a transmission electron microscope [16] or inferred from the 'return behaviour' in an STM [15], that the Au atoms, having formed a single-atom contact or atomic chain, collapse back into the surface after the rupture of the link. This results in a wider shape of the remaining tips, giving rise to thicker first contacts upon closing. Hansen et al [20] report a systematic study for contacts created with an STM at room temperature after different cleaning procedures of tip and surface. They find stable single-atom contacts less often when the contacts are free of contaminants such as, e.g., carbonates. We find a similar tendency, even stronger than in the low temperature measurements presented here, in lithographic break-junction experiments at room temperature under different vacuum conditions as well. As a rule of thumb the cleaner the conditions are at room temperature the more difficult it is to create stable, atomically sharp Au tips. However, once a single-atom contact is established, the steps in the closing curves are flat and close to integer values of $G_{0}$, presumably because the atoms have collapsed back to equilibrium positions. We will come back to this point in section 6 .

In figure 2 we show our results on $\mathrm{Zn}$ single-atom contacts (using the lithographic MCB technique [18]) with a preferred value of the single-atom contact of around $0.7-1.0 G_{0}$. Two more, but less pronounced maxima appear around $1.4 G_{0}$ and $2.5 G_{0}$. The histograms for opening and closing differ slightly; there is more weight at higher conductances but not as markedly as observed in Au. Yanson [21] found a similar histogram (only recorded for opening curves) for 'notched-wire' break-junctions [22] that have presumably less disorder in the contact region. The histogram of the sp-metal $\mathrm{Al}$ [23] (using again notched-wire break-junctions) shows well pronounced maxima with an average spacing of $1.2-1.3 G_{0}$ and a first maximum around $0.8 G_{0}$, thus at very similar positions

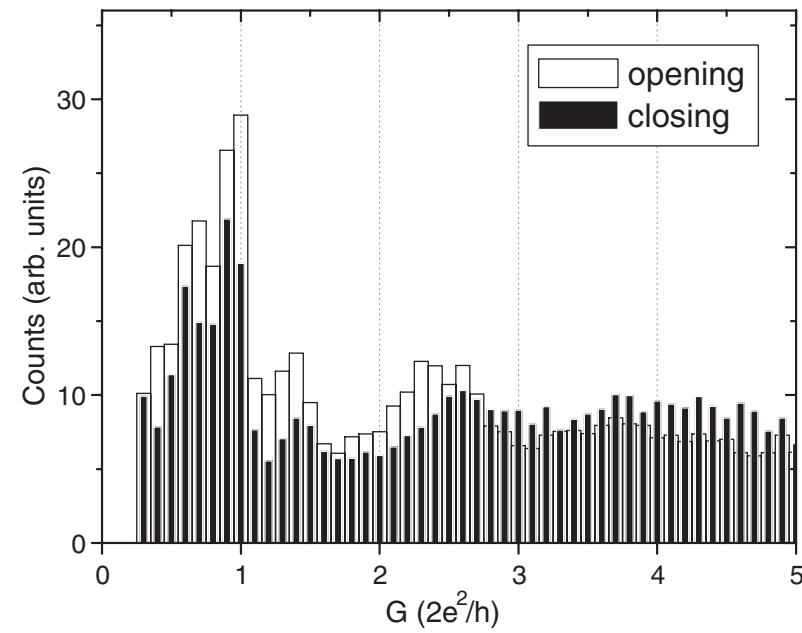

Figure 2. Conductance histogram of $\mathrm{Zn}$ lithographic break-junction, measured at $1.5 \mathrm{~K}$. The histograms have been calculated for $\sim 600$ individual opening and closing curves, respectively.
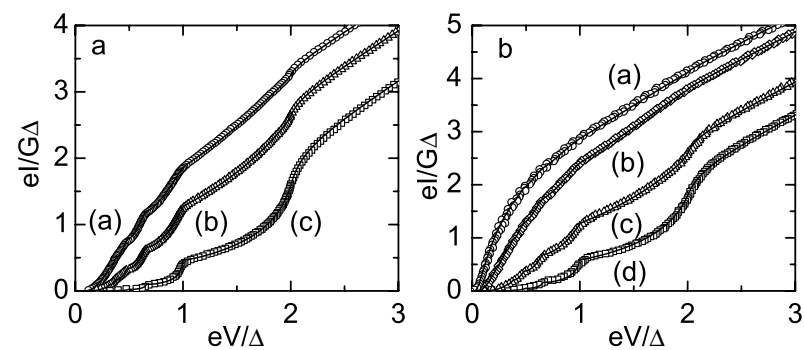

Figure 3. (A) Current-voltage characteristics of three different configurations with a conductance $G \simeq 0.8 G_{0}$ measured at $T<100 \mathrm{mK}$ on the same plateau of an opening experiment of an $\mathrm{Al}$ sample and best fits (curves) to the theory of MAR. The transmission coefficients are (a) $\tau_{1}=0.80, \tau_{2}=0.07$; (b) $\tau_{1}=0.68, \tau_{2}=0.12, \tau_{3}=0.02$; (c) $\tau_{1}=0.40, \tau_{2}=0.25, \tau_{3}=0.15$. (B) Current-voltage characteristics of four different configurations with a conductance $G \simeq 0.9 G_{0}$ measured at $T<270 \mathrm{mK}$ on a $\mathrm{Zn}$ whisker and best fits (curves) to the theory of MAR. The transmission coefficients are (a) $\tau_{1}=0.94, \tau_{2}=0.01$; (b) $\tau_{1}=0.86, \tau_{2}=0.01$; (c) $\tau_{1}=0.71, \tau_{2}=0.14, \tau_{3}=0.09$;

(d) $\tau_{1}=0.53, \tau_{2}=0.33, \tau_{3}=0.05$.

as $\mathrm{Zn}$. However, the histogram of $\mathrm{Zn}$ seems to feature more details. For instance, the first peak is split into two sub-peaks, presumably indicating two preferred contact configurations. The splitting is observed for histograms constructed from opening curves as well as from closing curves.

\section{Measurement of the transport channels}

For the determination of the transmission coefficients another observable has to be recorded, since the bare conductance only measures the sum of the coefficients. It has been shown previously that the 'channel ensemble' $\left\{\tau_{n}\right\}$ can be inferred from the current-voltage characteristics ( $I-V$ characteristics) of superconducting point contacts [24]. This is done by halting the elongation of the bridge at some point before breaking and recording $I-V \mathrm{~s}$ in the superconducting state of $\mathrm{Al}$ or other superconductors.

These experiments reveal different characteristics for contacts with the same total conductance and thus the same 
sum of $\tau_{n}$. As an example, figure 3(A) shows three $I-$ $V$ characteristics for contacts with $G \simeq 0.8 G_{0}$ recorded on the same 'plateau' of an opening curve of an $\mathrm{Al}$ sample but in different elongation states. The data have been recorded on a lithographic break-junction. Figure 3(B) displays three $I-V$ characteristics with $G \simeq 0.9 G_{0}$ for contacts from different, but consecutive opening procedures of a $\mathrm{Zn}$ whisker break-junction without closing the whisker thoroughly between the openings (for more details see [25]). The measuring temperature was $270 \mathrm{mK}$. Obviously, small changes of the configuration yield strong differences in the $I-$ $V$ characteristics and thus in the channel ensemble $\left\{\tau_{n}\right\}$ (see below). It is very likely that the observed changes in the $I-V$ characteristics are due to rearrangements in close vicinity of the central atom of the contact, because this part is most likely to be affected by the deformation of the bridge.

The strong current increases at voltage values $V=$ $2 \Delta / m e$ (where $m$ is an integer and $\Delta$ is the energy gap of the superconductor) are caused by multiple particle transport processes. Each current increase marks the onset of a new transport process with a different number $m$ of coherently transferred electron charges. The structure at $e V=2 \Delta$ corresponds to the transport of single electrons. Processes with $m>1$ are called multiple Andreev reflection (MAR). Their common factor is that the energy $2 \Delta$ has to be provided by the voltage source. Due to energy conservation, processes with higher $m$ set in at lower voltages since each quasiparticle transfer contributes the energy $e V$. The height of the current increase depends on the transmission of the individual channel since the probability of the simultaneous transport of $m$ electrons roughly follows the law $\tau_{n}^{m}[26,27]$.

This sets the basis for the determination of the channel ensemble $\left\{\tau_{n}\right\}$ in contacts with several channels. Because of the independence of the channels (eigenstates), the total current $I(V)$ through a contact with $N$ channels can be decomposed into the contributions of the individual channels [24]:

$$
I(V)=\sum_{n=1}^{N} \mathrm{i}\left(V, \tau_{n}\right) .
$$

The measured $I-V$ characteristics are fitted with the theory of MAR, with $N$ channels and $\left\{\tau_{n}\right\}$ as fitting parameters. The examples in figure 3 are described successfully with, at most, three channels (solid curves). Consequently, singleatom contacts of $\mathrm{Al}$ and $\mathrm{Zn}$ that contribute to the first maximum (around $0.8 G_{0}$ ) of the histograms accommodate up to three channels with different $\left\{\tau_{n}\right\}$. The analysis of a large number of those contacts reveals that for $\mathrm{Al}$ at least two channels contribute to the conductance on the last conductance plateau. However, in more than $80 \%$ of the contacts three channels have to be taken into account for a successful description of the superconducting $I-V$ characteristics [24]. For $\mathrm{Zn}$ samples in only a few cases three channels are present, most of the contacts transmit only one or two channels. A detailed discussion of the transport properties of $\mathrm{Zn}$ single-atom contacts will be published elsewhere [25]. Experiments with Au lithographic break-junctions (with $\mathrm{Al}$ leads for inducing superconductivity via the proximity effect) show a single channel in a single-atom configuration [28, 29].

\section{Conductance channels from atomic orbitals and transmission histograms}

The exact geometry of the contacts on the atomic scale is not known. However, it is very likely that the central part of the constriction consisting of the central atom and its direct neighbours is not a segment of a perfectly ordered single crystal. Because of the short Fermi wavelength the electrons feel all defects, such as voids, dislocations and impurity atoms, on this length scale. Therefore the eigenstates of this central part differ markedly from those of the leads, which are well described as Bloch waves. Due to the observed strong variation of the $\left\{\tau_{n}\right\}$ with the elongation, it is self-evident to start the solution of this problem with the linear combination of atomic orbitals (LCAO) [30, 31]. Two basic ingredients enter the model. The central atom has many fewer neighbours than an atom in the bulk. Consequently, the local band structure will differ from that of the bulk. The local Fermi energy will be shifted and has to be calculated self-consistently. Second, all electrons that are transferred via the constriction feel-apart from tunnel contributions - the valence orbitals of the central atom. Accordingly, the maximal number of channels of a single-atom contact is given by the number of the valence orbitals of the atom. The transmission coefficients of these channels depend on the overlap of the wavefunctions of the neighbouring atoms and thus on the exact atomic arrangement. The calculation for different metals in highly symmetric geometries (e.g. two fcc ordered pyramids facing each other and connected via a single atom) reveals that single-atom contacts of sp-like metals (Al, $\mathrm{Pb}$ ) with three p orbitals and one s orbital close to the Fermi energy, yields three channels with measurable transmission and a fourth one with a transmission lower than 0.01. The exact values of the $\tau_{n}$ do depend on the precise atomic configuration, but the number of channels does not. The monovalent metal $\mathrm{Au}$ accommodates one channel in agreement with the experimental findings.

According to simplistic arguments divalent elements should be insulating, since they have a completely filled outer s shell. This apparent discrepancy to the experimental findings indicates that additional orbitals besides the s orbitals contribute to the electronic conductance. Tight-binding calculations reveal that in the case of $\mathrm{Zn}$ the $4 \mathrm{p}$ orbitals are the most important ones for a correct description of the band structure [32]. With these considerations single-atom contacts of $\mathrm{Zn}$ are expected to have very similar transport properties as $\mathrm{Al}$ single-atom contacts, i.e. up to four channels with a total conductance of about $1 G_{0}$. The distribution of the transmission coefficients, however, may deviate, since it depends on the exact atomic configuration. Since $\mathrm{Zn}$ crystallizes in a hexagonal structure while Al is a fcc crystal, the actual atomic arrangement of the point contacts might be different. First calculations for $\mathrm{Zn}$ predict two channels for the pyramids oriented along the $c$-axis in very good agreement with our experimental findings. Further calculations are in progress [33].

In general, for contacts of multivalent metals none of the transmission coefficients achieves its saturation value $\tau_{n}=1$. This is not so much a consequence of atomic disorder but rather of the mismatch between the eigenstates of the contact region and the leads. A necessary condition for perfect transmission 
Mechanically controllable break-junctions for use as electrodes for molecular electronics
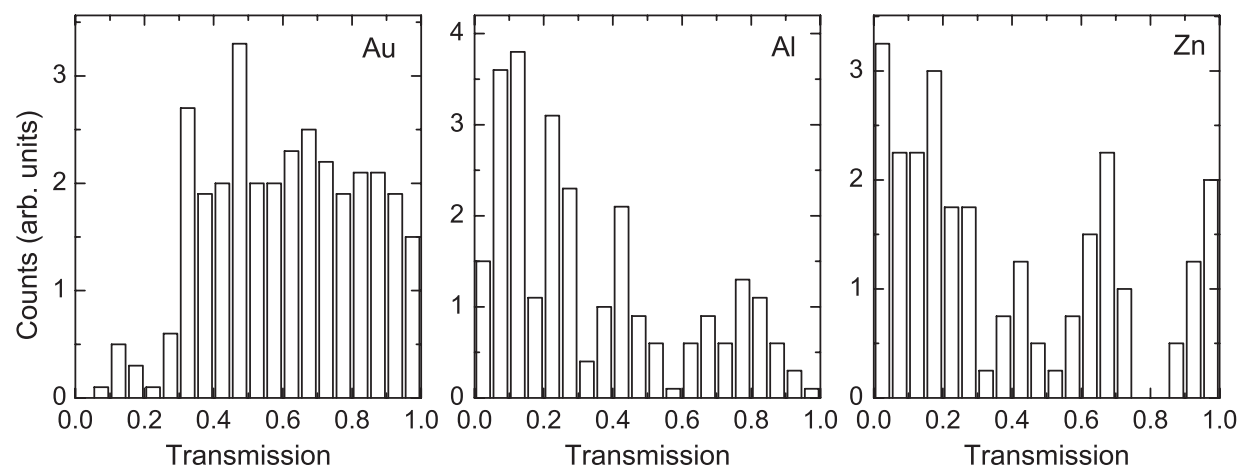

Figure 4. Left: transmission histogram for Au single-atom contacts, fabricated with the lithographic MCB technique. The histogram has been constructed from the analysis of the current-voltage characteristics of $\sim 350$ single-channel contacts of four different samples. Centre: transmission histogram for Al single-atom contacts, fabricated with the lithographic MCB technique. The histogram has been constructed from the analysis of the current-voltage characteristics of $\sim 250$ three-channel contacts of two different samples. Right: transmission histogram for $\mathrm{Zn}$ single-atom contacts, fabricated with the whisker and the notched-wire MCB technique. The histogram has been constructed from the analysis of the current-voltage characteristics of $\sim 100$ single-channel contacts of three samples.

is that the Fermi energy is located in the middle of the band. This is fulfilled for monovalent metals under the assumption of charge neutrality.

A second prerequisite for high transmission coefficients is a symmetric configuration of the central atom to its direct neighbours [34]. In the experiments on $\mathrm{Au}$, highly transmissive single-channel contacts are indeed observed [29], in particular after 'mechanically annealing' the sample, i.e. repeatedly opening and closing the contact. The latter procedure has been shown to be favourable for the formation of highly symmetric contacts of $\mathrm{Au}[35,36]$. Interestingly, single-atom contacts of $\mathrm{Zn}$ have a tendency to accommodate a very well transmitted single channel as well (see below).

As discussed before, for use in molecular electronics the transmission values might be interesting too. Therefore, we constructed transmission histograms for single-atom contacts of $\mathrm{Au}, \mathrm{Zn}$ and $\mathrm{Al}$ (see figure 4), i.e. contacts from last (first) plateaus of opening (closing) curves. Since the plateaus are often not clearly pronounced, we have to apply additional criteria in order to exclude larger than single-atom contacts. We therefore restrict the analysis to contacts with a maximum number of channels with measurable transmission, i.e. $\tau_{n}>$ 0.05 . This means that for Au we considered only singlechannel contacts, for $\mathrm{Zn}$ and $\mathrm{Al}$ we analysed contacts with up to three channels. For single-channel contacts of Au, it is easy to obtain stable contacts with transmission above $\tau \geqslant 0.3$. Contacts with lower transmission are found only occasionally. Above this threshold the distribution of transmission values is almost uniform. Apparently, among the three presented metals Au offers the best possibility to have highly transmissive channels. The average transmission of the investigated contacts is $\langle\tau\rangle=0.62$. When taking into account all contacts recorded on last or first plateaus with total conductance smaller than $1 G_{0}$ but allowing up to two channels, an average transmission of 0.8 is achieved. As stated before, this value might be even higher when using other contact fabrication methods than the lithographic MCB technique. The comparison between $\mathrm{Al}$ and $\mathrm{Zn}$ reveals that for $\mathrm{Zn}$ it is more likely to obtain very high transmission values above $\tau \sim 0.85$. In particular, it is possible to arrange stable contacts with one dominating channel and a much smaller second one (see curve (a) in figure $3(\mathrm{~B})$ ). This situation was achieved in $\mathrm{Al}$ contacts only for a short time when almost overstretching the contacts such that they broke shortly after. This observation has been explained by the formation of a resonant state for the dominating $\mathrm{sp}_{z}$ channel when the bonds of the central atom to its neighbours are nearly broken [37].

\section{First results of electronic transport through fullerene molecules}

While the physical properties, i.e. the possibility to form atomically sharp tips, might not be ideal for $\mathrm{Au}$; the good chemical properties make it the preferred material for contacting molecules, in particular at room temperature. Therefore we started with gold as electrode material for contacting fullerene molecules. We report here preliminary results about the electronic transport through the fullerene C60, obtained with the lithographic MCB technique on three, nominally identical samples. In the first experiment we evaporated the clusters under ultra-high vacuum (UHV) conditions with a pressure of about $10^{-7}$ mbar at room temperature onto an opened Au break-junction. The breakjunction has been prepared according to the method described by van Ruitenbeek et al [18]. After the final reactive ion etching step in oxygen plasma, that is supposed to successfully remove most organic residues, the sample is transferred into the UHV chamber where the bridge is broken repeatedly and $I-V$-characteristics are recorded in the single- or fewatom situation, i.e. with linear conductance close to $1 G_{0}$. Interestingly, most of the $I-V$ characteristics are nonlinear above approximately $0.2 \mathrm{~V}$ and show fluctuations of the conductance in the order of a few per cent. Typical traces are shown in figure 5. Similar behaviour has been reported recently by Hansen et al [20] in a study of Au single-atom contacts fabricated with an STM and has been attributed to contamination of the sample surface or the tip, respectively. Since it is not possible to clean the MCB, we have to keep in mind the possible presence of contaminating molecules or atoms when interpretating the data with the fullerene molecules. At voltages of typically $0.5-0.6 \mathrm{~V}$ the contacts break. When withdrawing the pushing rod of the bending mechanism, a new contact can be formed. Amelioration of the 


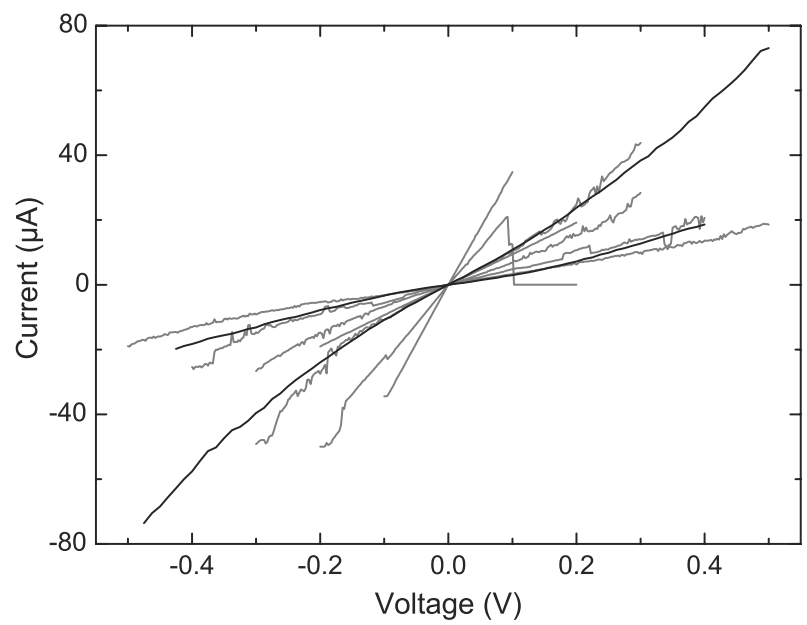

Figure 5. Current-voltage characteristics of few-atom contacts of an Au break-junction at room temperature under UHV conditions. Grey curves: before (black curves: after) evaporation of C60 clusters.

vacuum conditions to a pressure of $p<10^{-8}$ mbar did not change the principal behaviour of the contacts. However, it became less likely to arrange contacts with a conductance as small as $1 G_{0}$ as discussed in section 3 .

After these tests the bridge is opened to an electrode distance of about $1 \mathrm{~nm}$ corresponding to the diameter of a C60 cluster. The fullerene clusters are evaporated while monitoring the resistance of the bridge with a constant voltage of $0.2 \mathrm{~V}$. Due to thermal expansion of the electrodes when heating the evaporation crucible, located only $5 \mathrm{~cm}$ below the sample, the resistance is not constant, but has a tendency to decrease. Therefore, the bending mechanism is adjusted in order to keep the distance of the electrodes constant. The temperature of the crucible is kept slightly below the evaporation temperature of C60 until thermal equilibrium is reached, i.e. the resistance remains constant. No further resistance change is observed when the evaporation starts. Thus, we do not detect whether a molecule is captured between the electrodes. After the evaporation of a thickness of a few nanometres, the evaporation is stopped, the electrodes are brought back into contact, and $I-V$ characteristics are recorded. Two examples of $I-$ $V$ characteristics with $\mathrm{C} 60$ evaporated onto the electrodes are shown in figure 5 as black lines. Interestingly, high conductance values of the order of $1 G_{0}$ (at low bias) are often observed. The characteristic nonlinearity of the $I$ $V$ characteristics of the Au electrodes before evaporation of the clusters remains unchanged as well. The presence of the fullerenes shows up as an apparent stabilization of the contacts, i.e. the fluctuations of the conductance are reduced and the contacts sustain larger voltages of up to $1 \mathrm{~V}$. Thus, it is unclear whether the measuring current did indeed flow through one (or several) C60 molecule(s) or whether, in fact, a $\mathrm{Au}-\mathrm{Au}$ contact, stabilized by carbon, has been investigated. In order to obtain further insight, the bridge was broken again and the evaporation and measurement procedure was repeated several times without any change of the observations. After evaporation of approximately $180 \mathrm{~nm}$ of C60 total thickness onto the Au break-junction with an initial thickness of $100 \mathrm{~nm}$, the sample was brought back to ambient conditions and the

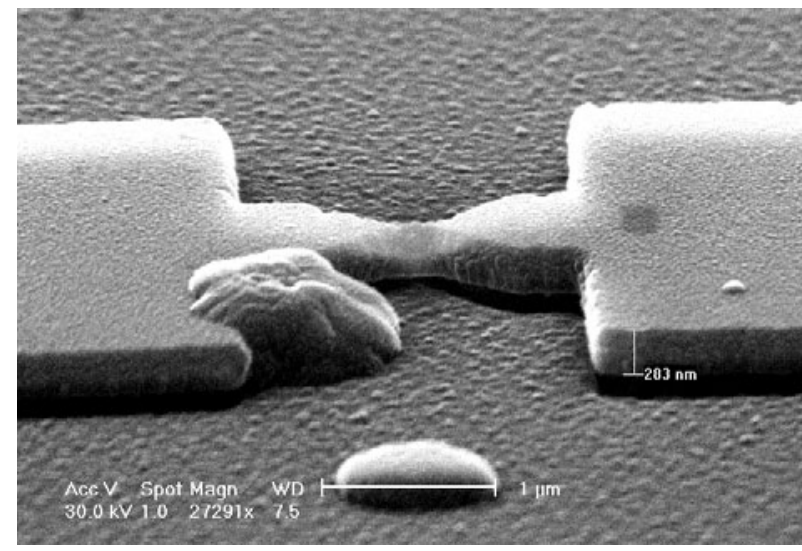

Figure 6. Electron micrograph of the sample (after measurement) with approximately $180 \mathrm{~nm}$ C60 evaporated on top of the Au layer with an initial thickness of $100 \mathrm{~nm}$. The $I-V$ characteristics which are depicted in figure 5 have been recorded on this sample.

electron micrograph figure 6 was taken. Although it is very likely that electron transport had taken place through C60 it cannot be ruled out that a direct contact between the Au electrodes was formed. In an improved experiment with a larger distance between the crucible and sample the heating and thermal expansion of the electrodes was avoided, but the same observations were made for the $I-V$ characteristics and the preferred linear conductance values. We thus conclude that C60 between Au electrodes has the tendency to form contacts with conductance values close to $G_{0}$. However, the number of contributing channels is not clear yet. To the best of our knowledge, no calculations have been performed so far for this system. However, several groups have studied the transport through a $\mathrm{C} 60$ cage contacted by $\mathrm{Al}$ electrodes $[38,39]$. They find conductance values in the order of a few tenths of $G_{0}$ up to $3 G_{0}$ transmitted by up to three transport channels depending on the contact geometry and a possible potential shift upon applying a gate voltage.

In a further experiment we therefore fabricated a breakjunction without Au electrodes by evaporating $700 \mathrm{~nm}$ of C60 directly onto a lithographic mask. After the evaporation the sample is taken out of the vacuum and the fabrication procedure is accomplished as usual, i.e. a lift-off step in acetone for removing the mask and a dry etching step in oxygen plasma are performed. A first sample fabricated by this method showed a resistance of approximately $200 \mathrm{M} \Omega$ before break and similar nonlinear $I-V$ characteristics as observed in the experiment with the Au electrodes. Experiments with $\mathrm{Al}$ electrodes at room temperature as well as at low temperature are in progress.

\section{Conclusions and outlook}

As a prerequisite for the use of break-junction electrodes for contacting individual nanoobjects a characterization scheme of the transport properties of electrodes has been developed. We have demonstrated that the noble metal Au as well as the divalent metal $\mathrm{Zn}$ offers the possibility to form contacts with a well-transmitted single transport channel. Of course, these findings give no answer to the question of how the transmission through the coupled system consisting of electrode, nanoobject and electrode is accomplished. However, it can be expected that the number of contributing channels will not be higher 
than the maximum that can be transferred by the tip atom of the electrode themselves. Smit et al [8] found that the number of channels of Pt electrodes was reduced to a single, almost perfectly transmissive channel when capturing a hydrogen molecule between the tips. The mechanical properties, i.e. the ability to form atomically sharp tips, is the second important ingredient for contacting individual nanoobjects. In this respect the noble metal Au with its tendency to form smooth surfaces seems not to be the ideal choice. On the other hand, because of its good chemical properties it is still one of the most commonly used materials.

A major drawback of the MCB technique is the difficulty of adding gate electrodes to the circuit. Because of the relatively large amount of metal around the contacted object and the large distance to the substrate the electric fields of backgates are essentially screened. The development of closeby gates on the electrodes themselves is in progress.

\section{Acknowledgments}

We thank our colleagues W Belzig, J C Cuevas, G Ganteför, B Kessler, A Levy Yeyati, P Leiderer, P Nielaba, J van Ruitenbeek and $\mathrm{C}$ Urbina for many fruitful discussions and our co-workers V Kunej and H-F Pernau for their contributions to the work presented here. Financial support by the Alfried Krupp von Bohlen und Halbach foundation and the Deutsche Forschungsgemeinschaft is gratefully acknowledged.

\section{References}

[1] Park H, Lim A K L, Alivisatos A P, Park J and McEuen P L 1999 Appl. Phys. Lett. 75 301-3

[2] Morpurgo A F, Marcus C M and Robinson D B 1999 Phys. Rev. Lett. 74 2084-6

[3] Joachim C, Gimzewski J K, Schlitter R R and Chavy C 1995 Phys. Rev. Lett. 74 2102-5

[4] Reed M A, Zhou C, Muller C J, Burgin T P and Tour J M 1997 Science 278 252-4

[5] Kergueris C, Bourgoin J P, Palacin S, Esteve D, Urbina U, Magoga M and Joachim C 1999 Phys. Rev. B 59 12505-13

[6] Reichert J, Ochs R, Beckmann D, Weber H B, Mayor M and Löhneysen H v 2002 Phys. Rev. Lett. 88176804

[7] Nitzan A 2001 Annu. Rev. Phys. Chem. 52 681-750

[8] Smit R H M, Noat Y, Untiedt C, Lang N D, van Hemert M C and van Ruitenbeek J M 2002 Nature 419 906-8

[9] Landauer R 1970 Phil. Mag. 21 863-7

[10] Büttiker M 1986 Phys. Rev. Lett. 57 1761-4

[11] Datta S 1995 Electronic Transport in Mesoscopic Systems (Cambridge: Cambridge University Press)

[12] Devoret M H and Grabert H (ed) 1992 Single Charge Tunneling (NATO ASI Series B294) (New York: Plenum)
[13] Dorokhov O N 1984 Solid State Commun. 51 381-4

[14] Agraït N, Levy Yeyati A and van Ruitenbeek J M 2003 Quantum properties of atomic-sized conductors Phys. Rep. $37781-279$

[15] Yanson A I, Rubio Bollinger G, van den Brom H E, Agrait N and van Ruitenbeek J M 1998 Nature $395783-5$

[16] Ohnishi H, Kondo Y and Takayanagi K 1998 Nature 395 780-3

[17] Costa-Krämer J L, Garcia N, Garcia-Mochales P and Serena P A 1995 Science 342 1144-9

[18] van Ruitenbeek J M, Alvarez A, Piñeyro I, Grahmann C, Joyez P, Devoret M H, Esteve D and Urbina C 1996 Rev. Sci. Instrum. 67 108-11

[19] Brandbyge M, Sœrensen M R and Jacobsen K W 1997 Phys. Rev. B 56 14956-9

[20] Hansen K, Nielsen S K, Brandbyge M, Lægsgaard E, Stensgaard I and Besenbacher F 2000 Appl. Phys. Lett. 77 708-10

[21] Yanson A I 2001 Atomic chains and electronic shells: quantum mechanisms for the formation of nanowires $P h D$ Thesis University of Leiden, The Netherlands

[22] Muller C J et al 1992 Physica C 191 485-92

[23] Yanson A I and van Ruitenbeek J M 1997 Phys. Rev. Lett. 79 2157

[24] Scheer E, Joyez P, Esteve D, Urbina C and Devoret M H 1997 Phys. Rev. Lett. 78 3535-8

[25] Konrad P, Brenner B, Bacca C, Löhneysen H v and Scheer E 2004 Appl. Phys. Lett. submitted

[26] Averin D and Bardas A 1995 Phys. Rev. Lett. 75 1831-4

[27] Cuevas J C, Martín-Rodero A and Levy Yeyati A 1996 Phys. Rev. B 54 7366-79

[28] Scheer E, Agraï N, Cuevas J C, Levy Yeyati A, Ludoph B, Martín-Rodero A, Rubio Bollinger G, van Ruitenbeek J M and Urbina C 1998 Nature 394 154-7

[29] Scheer E, Belzig W, Naveh Y, Devoret M H, Esteve D and Urbina C 2001 Phys. Rev. Lett. 86 284-7

[30] Cuevas J C, Levy Yeyati A and Martín-Rodero A 1998 Phys. Rev. Lett. 80 1066-9

[31] Levy Yeyati A, Martín-Rodero A and Flores F 1997 Phys. Rev. B 56 10369-72

[32] Papaconstantopoulos D A 1986 Handbook of the Band Structure of Elemental Solids (New York: Plenum)

[33] Häfner M, Heurich J and Cuevas J C 2003 unpublished

[34] Heurich J 2003 Elektronischer Transport durch Einzelatom- und Einzelmolekülkontakte Thesis Universität Karlsruhe

[35] Agraït N, Rodrigo J G, Sirvent C and Vieira S 1993 Phys. Rev. B 48 8499-501

[36] Untiedt C, Rubio G, Vieira S and Agraït N 1997 Phys. Rev. B $562154-60$

[37] Cuevas J C, Levy Yeyati A, Martín-Rodero A, Rubio Bollinger G, Untiedt C and Agraït N 1998 Phys. Rev. Lett. 81 2990-3

[38] Palacios J J, Pérez-Jiménez A J, Louis E and Vergés J A 2001 Nanotechnology 12 160-3

[39] Taylor J, Guo H and Wang J 2001 Phys. Rev. B 63121104 